\title{
Fabrication and Analogue Applications of nanoSQUIDs using Dayem Bridge Junctions
}

\author{
L. Hao, D.C. Cox, J.C. Gallop, J. Chen Member IEEE
}

\begin{abstract}
We report here recent work at the UK National Physical Laboratory (NPL) on developing nanoscale SQUIDs using Dayem bridge Josephson junctions. The advantages are simplicity of fabrication, exceptional low-noise performance, towards the quantum limit, and a range of novel applications. Focused ion beam patterned Nb SQUID, possessing exceptionally low noise $\left(\sim 200 \mathrm{n} \Phi_{0} / \mathrm{Hz}^{1 / 2}\right.$ above $\left.1 \mathrm{kHz}\right)$, and operating above $4.2 \mathrm{~K}$ can be applied to measurement of nanoscale magnetic objects or coupled to nano-electromechanical (NEMS) resonators, as well as single particle detection of photons, protons and ions. The limited operating temperature range may be extended by exposing the Dayem bridges to carefully controlled ion beam implantation, leading to non-reversible changes in junction transition temperature.
\end{abstract}

Index Terms - NanoSQUIDs, nanomagnetism, NEMS Mechanical Resonators. Single particle detection.

\section{INTRODUCTION}

It has been appreciated for almost 50 years that SQUIDs (Superconducting QUantum Interference Devices) are macroscopic quantum objects which are capable of detecting and measuring a wide range of physical parameters with unequalled sensitivity. Magnetic flux changes are the natural, most straightforward quantity to which a SQUID is sensitive but spatial displacement, particle detection and mass sensing characterize the range of other physical parameters which SQUIDs are capable of measuring with unparalleled sensitivity. For comprehensive reviews of many aspects of SQUID theory and technology see [1]. Although the first SQUIDs that demonstrated an approach to the standard quantum limit were of relatively large size (typically one tenth of millimetre or so in linear dimension) there is no intrinsic reason why smaller SQUIDs should not also approach this level of sensitivity. In addition SQUIDs which approach the nanoscale in size will open up sensitive measurement of a new range of quantities [2-4]. In this paper we describe fabrication methods which we have developed at NPL to realize nanoSQUIDs, then go on to outline the performance achieved to date. Finally we describe some applications and speculate about future developments [5].

\section{DEVICE FABRICATION}

\section{A. NanoSQUIDs require different Josephson junctions}

A SQUID is not generally used as an object in isolation. In measurement and detection applications an input transducer is required to convert a physical quantity of interest to magnetic

Ling Hao, John Gallop and David Cox are with the Quantum Detection Group, National Physical Laboratory, Teddington, TW11 OLW, (UK). Jie Chen is in the Department of Mechanical Engineering in the School of Engineering and Design at Brunel University, Uxbridge, UB8 3PH, (UK) flux and design of this transduction process is crucial to achieving optimal performance. To couple efficiently it is often desirable to match the size of the SQUID coupling coil to the scale of the system to be measured. Although in principle an intermediate superconducting flux transformer is usual, in practice this is not feasible at the sub-micron level. Multi-turn, sub-micron coils are not yet routinely attainable, and in addition the stray inductance arising from the leads connecting primary to secondary will make such a transformer far from optimal. Thus the present tendency is to use direct coupling between NEMS resonator and nanoSQUIDs.

Direct coupling between object to be sensed and the SQUID requires that the overall size of the SQUID loop should be sub-micron. This in turn places even more stringent requirements on the dimensions of the junctions. There are major difficulties in using conventional tri-layer Josephson tunnel junctions at sub-micron scale, particularly when operated at temperatures $T$ around $4 \mathrm{~K}$ or above. The high operating temperature requires that the junction critical current $I_{c} \gg k_{B} T / \Phi_{0}$ (where $k_{B}$ is Boltzmann's constant and $\Phi_{0}$ is the flux quantum $h / 2 e$ ) implying critical currents $I_{c} \gg 1 \mu \mathrm{A}$. Josephson tunnel junctions of size, say $50 \mathrm{~nm}$ square size, require critical current densities as high as $10^{9} \mathrm{~A} / \mathrm{m}^{2}$ to attain such critical currents. Current trilayer insulating barrier technologies typically only achieve critical current densities some two orders of magnitude less than this and junctions any larger than $50 \mathrm{~nm}$ square would be incompatible with submicrometre loop dimensions of the nanoSQUID. To deal with this dilemma at NPL we have pursued a different junction technology, using Dayem or weak-link 'nano-bridge' junctions prepared by focused ion beam (FIB) milling of a thin superconducting film. The resulting junctions are only around $65 \mathrm{~nm}$ in length and width [6] and have the added advantage of operating in higher applied fields than trilayer junctions. Other groups have approached this nanoSQUID requirement from a different fabrication direction. One technique [7], using trilayer methods based on superconductor-normalsuperconductor (SNS) junctions, provides an alternative means for achieving high critical current densities, though the junction sizes achieved are not yet as small as Dayem bridge designs. A second approach [8] uses conventional oxide barrier Josephson junctions but, through an interesting use of FIB milling, is able to provide flux capture areas of $0.2 \mu \mathrm{m}^{2}$ and total junction area of similar size. 


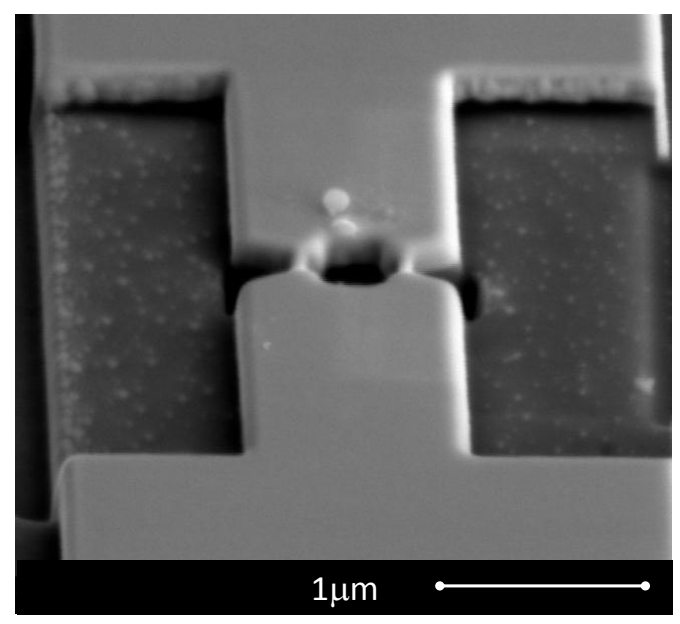

Fig. 1 SEM image of $\mathrm{Nb}$ nanoSQUID fabricated by FIB milling. The loop diameter is around $350 \mathrm{~nm}$, each junction is around $65 \mathrm{~nm}$ in length. A magnetic particle $100 \mathrm{~nm}$ in diameter is placed on the loop perimeter.

\section{B. Focused Ion Beam (FIB) Milling of Nanobridge SQUID}

Initially a niobium $(\mathrm{Nb})$ thin film is sputter-deposited to a thickness of approximately $150-200 \mathrm{~nm}$ on a $\mathrm{Si}$ substrate. Optical lithography is then used to pattern a set of strips (around $5 \mu \mathrm{m}$ wide) in the resulting thin film. Also larger bond-pads, overlaying $\mathrm{Au}$ pads for wire-bonding, are patterned. The patterned chip (typically $10 \mathrm{~mm} \times 10 \mathrm{~mm}$ in size and $0.5 \mathrm{~mm}$ thick) is transferred to the FEI dual-beam FIB system. As we consider in more detail below, it is important to consider the influence of the focused ions on the $\mathrm{Nb}$ film. Consequently a thin layer of amorphous tungsten is first deposited using e-beam decomposition of $\mathrm{W}(\mathrm{CO})_{6}$. This has two roles; first it provides protection for the $\mathrm{Nb}$ film against $\mathrm{Ga}$ ion poisoning by implantation during the FIB milling process. Second since this tungsten layer has metallic behavior (although with rather high resistivity) it provides a normal metal shunt resistance path in parallel with the $\mathrm{Nb}$ film.

The focused Ga ion beam is first used to pattern a circular or rectangular hole within one of the $\mathrm{Nb}$ strips which forms the SQUID loop. Next the beam is used to mill away the bilayer to provide two nanobridges within the pre-patterned SQUID loop. These are typically $60-80 \mathrm{~nm}$ in both width and length, whereas the film thickness in the milled-down region is around $50 \mathrm{~nm}$. A scanning electron microscope (SEM) image of a typical device is shown in figure 1. Devices made in this way show a high level of reproducibility and we have shown them to be very stable in their current voltage characteristics over periods of years when stored at room temperature in a dessicator. Note that these dimensions represent the overall size of the junctions but, due to $\mathrm{Ga}$ implantation and other damage to the film surface this is only an upper limit on the cross sectional area and length of a junction. It is interesting that these dimensions are on the same order or rather less than the temperature dependent coherence length for bulk $\mathrm{Nb}$ over the operational temperature range. It is for this reason that we believe these nanoSQUIDs show classical Josephson properties.

\section{Nb/Ga-ion interactions}

It is worth considering the expected influence of the FIB milling process on the properties of the Josephson junctions and SQUID ring which may arise from modifications due to impact of Ga ions. The interaction of high-energy ions with a solid substrate gives rise to implantation of the ion species and sputtering of the target material. In our case the typical beam energy is $30 \mathrm{keV}$, where the optics of ion columns are optimised to produce the smallest beam spot. In general terms the implant depth of the ions and the sputter yield (atoms/ion) scale with the mass of the target species. According to calculations using SRIM [8] software irradiating $\mathrm{Nb}$ with a $\mathrm{Ga}$ FIB at $30 \mathrm{keV}$ results in ion sputter yields of almost 4 atoms/ion with a mean implant depth around $12 \mathrm{~nm}$ whereas the maximum possible implant depth approaches $40 \mathrm{~nm}$. Our experimental evidence for milling rates is in good agreement with the SRIM predictions.

In addition to implantation and sputtering a SRIM prediction can be made of induced damage from the ion beam. At our expected ion dose SRIM predicts 630 vacancies per ion resulting in well in excess of $10^{9}$ vacancies generated in the implanted volume. This should be high enough to amorphise the $\mathrm{Nb}$ lattice in the exposed region, but in reality much of this damage would heal during exposure due to further ion collisions. Nevertheless such damage would be expected to lead to significant changes in the local $\mathrm{Nb}$ superconducting properties. In the next section we show that all superconductivity is lost if the ion dose is high enough, and, importantly for wider applications of nanoSQUIDs, the superconducting transition temperature $T_{c}$ may be suppressed by careful application of ion beam exposure.

\section{Suppression of transition temperature.}

We will see in subsequent discussions of the FIBbed nanoSQUID noise performance that the properties of these devices are nearly ideal. The only limitation of which we are aware is that their operating temperature range is relatively narrow in the as-prepared state. Using $\mathrm{Nb}$ as a superconductor the standard SQUIDs made this way operate between $6.5 \mathrm{~K}$ and $8 \mathrm{~K}$. Although for some uses this is advantageously above $4.2 \mathrm{~K}$, for others it would be better to be able to suppress and/or extend the operating range.

Following on from the discussion of the preceding section we have attempted to tune the operating temperature by controlled focused ion beam dosage of the SQUID films. To date this has involved measuring the transition temperatures and resistivities of a number of $\mathrm{Nb}$ thin film strips, subject to each receiving different controlled exposure to the beam. Small areas across the entire width of a $4 \mu \mathrm{m}$ wide track of $\mathrm{Nb}$ were scanned by a FIB beam at a current of $100 \mathrm{pA}$, with steps in both $x$ and $y$ axes of $10 \mathrm{~nm}$. At each site the dwell time was $1 \mu \mathrm{s}$, resulting in $6.25 \times 10^{6}$ ions per $\mu \mathrm{m}^{2}\left(30 \mu \mathrm{C} / \mathrm{cm}^{2}\right)$ for every pass of the beam. In fig. 2 we show how the increasing 


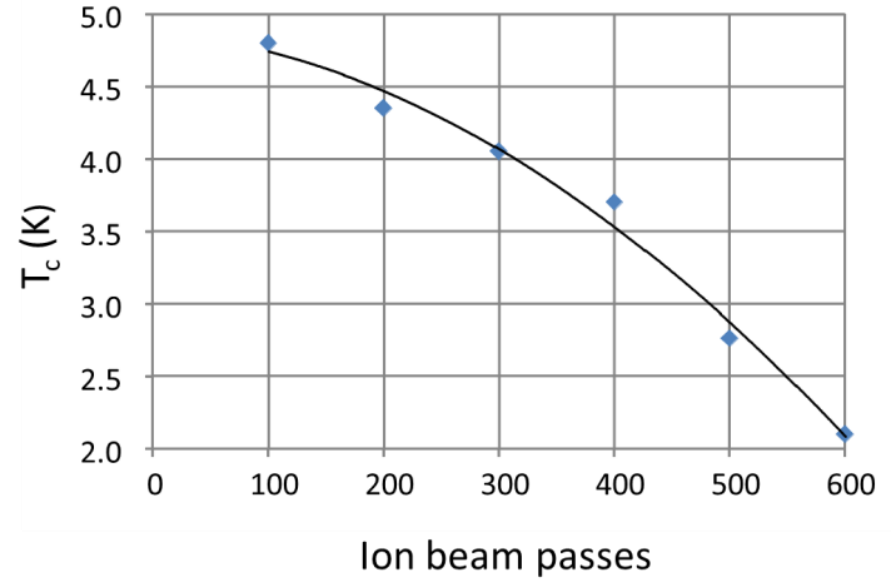

Fig. 2. Suppression of transition temperature $T_{c}$ by controlled ion beam dose. The fitted curve is a quadratic.

number of passes applied to the strips results in suppression of the strip transition temperature. This result shows promise for future design of FIBbed nanoSQUIDs with tailored operating temperatures.

\section{CHARACTERISATION AND NOISE PERFORMANCE OF FIBBED NANOSQUIDS}

\section{A. Current Voltage Characteristics and their Temperature Dependence}

A wide range of SQUID loop sizes have been fabricated by the method described above, with loop dimensions from $100 \mathrm{~nm} \times 1 \mu \mathrm{m}$ to $50 \mu \mathrm{m} \times 50 \mu \mathrm{m}$. For all designs the current voltage characteristics (IVCs) have essentially the same features, showing classic resistively shunted junction (RSJ) behavior over a range of temperatures of around $1.5 \mathrm{~K}$ below the junction transition temperature (see Fig. 3). At temperatures below this hysteretic behaviour begins, which we attribute to hot-spot generation, following the descriptions given in a number of references, see for example [10-12]. The condition for non-hysteretic behavior is that the re-trapping current $I_{r}$, that is the minimum current needed to sustain a normal state hotspot once $I_{c}$ has been exceeded, must be smaller than $I_{c}$ itself.

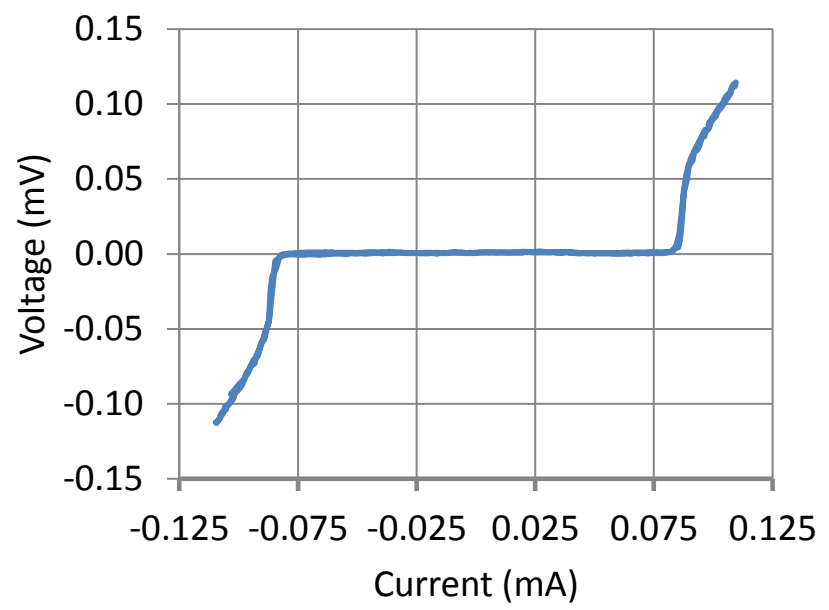

Fig. 3 shows a current voltage characteristic (IVC) for a particular nanoSQUID with loop size $350 \mathrm{~nm}$ at a temperature of $7.0 \mathrm{~K}$.

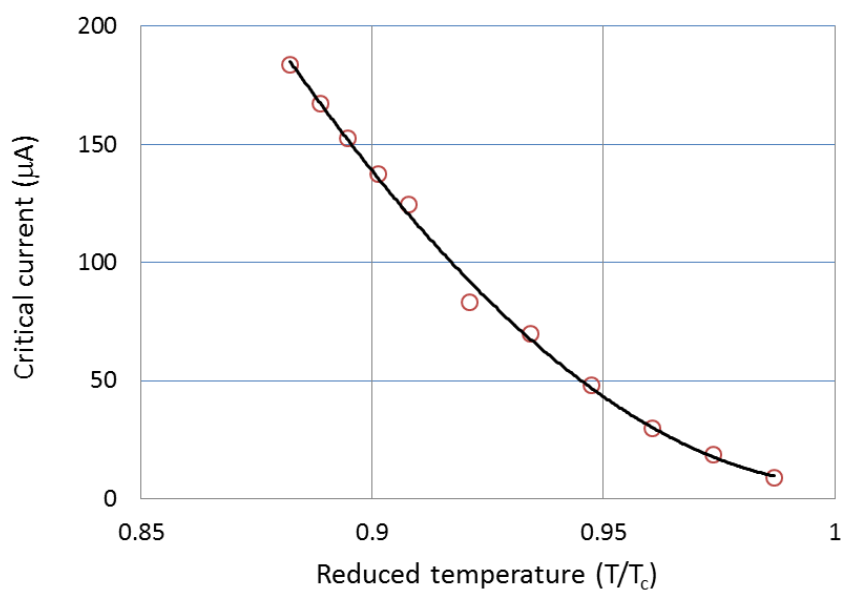

Fig. 4. Temperature dependence of critical current as a function or reduced temperature $\left(T / T_{c}\right)$. The solid line is a fit to the relationship $I_{c}(T) \alpha\left(1-\left(T / T_{c}\right)\right)^{2}$ with $T_{c}=7.73 \mathrm{~K}$.

Fig.4 shows the measured variation of critical current of a typical Dayem bridge Nb nanoSQUID with temperature for the non-hysteretic regime. This shows a temperature dependence which is of the form

$$
I_{c}=I_{0}\left(1-\left(T / T_{c}\right)\right)^{2}
$$

This behavior is compatible with the theoretical dependence of a weak link short compared with the Ginzburg-Landau coherence length $\xi(T)$. This condition would be satisfied for our junctions if the values for $\xi(T) \sim 40-70 \mathrm{~nm}$ for bulk $\mathrm{Nb}$ in the clean limit (electronic mean free path comparable with $\xi(T))$ are valid.

The optimum biasing condition for a d.c. SQUID when read out by a following voltage preamplifier is generally when the mean output voltage level $V$ coincides with the maximum value for the differential resistance $\rho=(d V / d i)$ of the nanoSQUID is a maximum. Note that the flux to voltage transfer function $\mathrm{d} V / \mathrm{d} \Phi$ measured at the SQUID voltage output can be as high as $3.6 \mathrm{mV} / \Phi_{0}$ for our devices. A slightly non-linear dependence of flux periodicity on field is seen in all of our nanoSQUIDs and is believed to arise from a combination of varying penetration depth with field and flux focusing. This is particularly noticeable for nanoSQUIDs since relatively high values of applied magnetic field (as much as $10 \mathrm{mT}$ ) are required to produce a change of one $\Phi_{0}$ in applied flux. At relatively high operating temperatures this field level is sufficient to slightly alter the superconducting penetration depth.

The flux noise originating in several of these nanoSQUID designs has been measured as a function of frequency from $0.1 \mathrm{~Hz}$ to $3 \mathrm{kHz}$. Initial use of a low noise room temperature preamplifier with white noise referred to the input of $1.6 \mathrm{nV} / \mathrm{Hz}^{1 / 2}$ showed that preamplifier noise limited the SQUID noise, since the differential resistance at the operating point was typically only a few ohms or less. To reduce the effect of preamplifier noise we have subsequently used a series SQUID array (SSA) amplifier [13], operated at 4.2K. 
This is a current sensing amplifier with a white noise floor of $10 \mathrm{pA} / \mathrm{Hz}^{1 / 2}$ and is operated as shown in the schematic circuit diagram (Fig. 5).

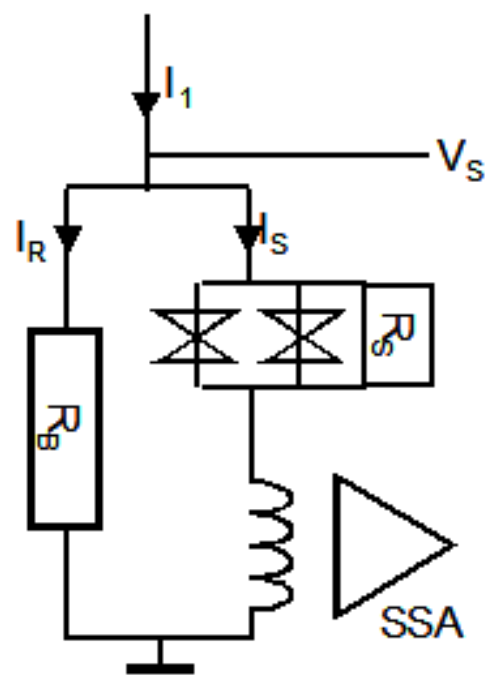

Fig. 5 Schematic of nanoSQUID bias and readout circuit showing the series SQUID array (SSA) input connection to the nanoSQUID.

The SSA preamplifier is held at $4.2 \mathrm{~K}$ in good thermal contact with a liquid helium bath while the nanoSQUID is controlled at a selected higher temperature with a stability of around $0.1 \mathrm{mK}$. A small superconducting solenoid is provided to apply a magnetic field perpendicular to the plane of the nanoSQUID, to investigate the periodic flux response and to allow optimization of the operating conditions.

The $\mathrm{Nb}$ nanoSQUIDs measured to date show remarkably low flux noise [6] and can operate in high ambient magnetic fields [14]. It is also clear that the white noise level still contains a significant contribution from the SSA preamplifier, particularly at low frequencies, below $\sim 1 \mathrm{kHz}$. Further work is underway to optimize the properties of our devices since this will reduce the influence of the noise originating within the SSA amplifier.

\section{OPTIMISED COUPLING TO SQUID SERIES ARRAY AMPLIFIER}

The performance of the nanoSQUID when coupled to the current sensing input of the SSA is optimized by rather different parameters to those required for direct voltage readout via a room temperature voltage amplifier. In the case of low frequency signals the most important parameter is the output current to flux transfer function $g=d I_{s} / d \Phi$ which depends both on maximizing the critical current value (while avoiding any hysteretic behaviour), ensuring the critical current values of the individual junctions are matched as well as possible and finally that the McCumber parameter $\beta_{L}=2 L I_{c} / \Phi_{0} \leq 1$. The latter ensures that the flux modulation depth $\Delta I$ of the critical current of the two junctions in parallel is greater than 0.5 (where $\Delta I$ is a dimensionless parameter defining the fractional range of critical current modulation produced by the applied flux.).
Estimation of the transfer function $g$ is done by first solving the three simultaneous equations below which describe the currents through each arm of the circuit in terms of the circuit components of the SQUID and the bias resistor $R_{B}$ (see figure 5).

$$
\begin{aligned}
& V_{s}\left(I_{s}\right)=R_{B} I_{R} \\
& I_{1}=I_{R}+I_{s} \\
& V_{s}\left(I_{s}\right)=R_{s} I_{c}\left[\left(\frac{I_{s}}{I_{c}}\right)^{2}-1\right]^{1 / 2}
\end{aligned}
$$

where equation (4) has the solution $V_{s}\left(\left|I_{s}\right|<I_{c}\right)=0$ whenever the bias current $I_{s}<I_{c}\left(\Phi_{x}\right)$. The form of equation (4) assumes that the low capacitance RSJ model is a good description of our microbridge junctions' current voltage characteristics which proves to be the case for the vast majority of our Dayem bridge devices. This also means that the junctions have current-phase relationships which are well described by a simple sinusoidal dependence. In the light of this the SQUID critical current will be dependent on the applied flux $\Phi_{x}$, with approximate functional variation of the form

$$
I_{c}\left(\Phi_{x}\right)=I_{c 0}\left[(1-\Delta I)+\Delta I \cos \left(\pi \Phi_{x}\right)\right]
$$

Solving these equations for the variation of current $I_{s}\left(\Phi_{x}\right)$ yields the behavior shown in fig. 6 for a range of both $I_{c 0}$ and $\Delta I$ values. Note the flux quanta periodic behavior provided the nanoSQUID current voltage characteristic is single valued.

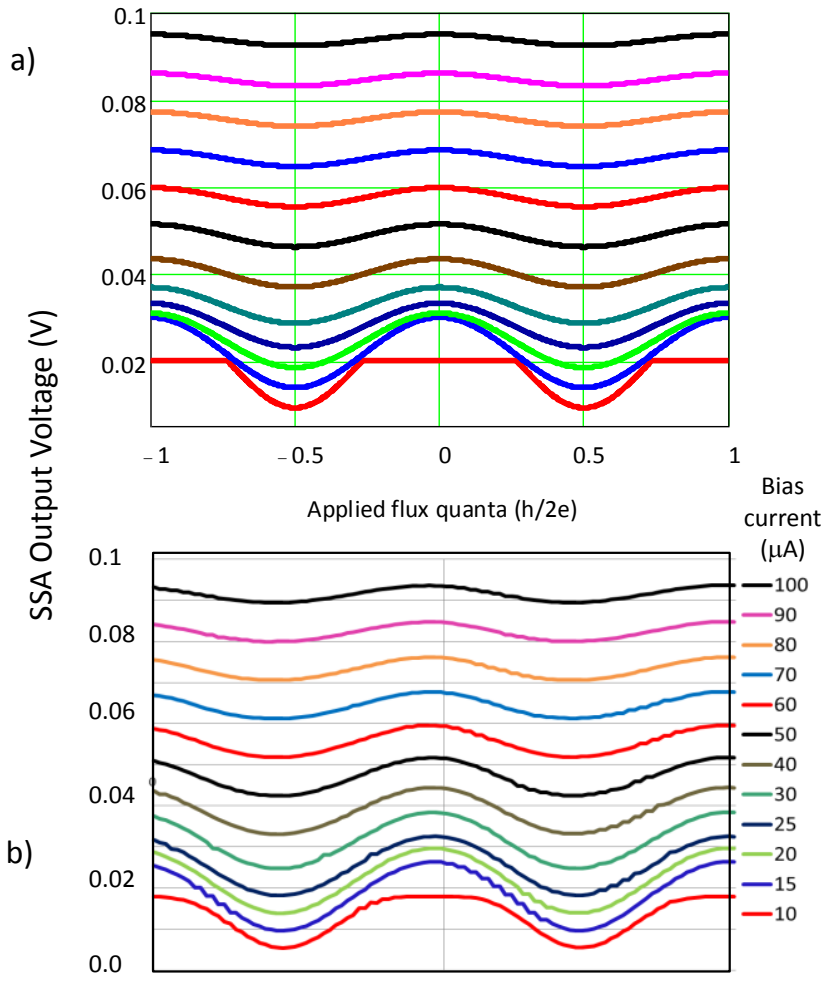

Fig. 6 a) Calculated and b) experimental SSA output voltage for a range of applied flux for a $\mathrm{Nb}$ nanoSQUID and a set of bias currents, ranging from $10 \mu \mathrm{A}$ to $100 \mu \mathrm{A}$. The best fit values are $I_{c 0}=15 \mu \mathrm{A}, R_{s}=0.5 \Omega$ and $\Delta I=0.9$. 
Our model allows us to calculate the input flux to current transfer function $g$ for the nanoSQUID. This depends on the applied magnetic flux and to optimise $g$ it is necessary to select a suitable working point value for $\Phi_{x}$. Maximum values of $g$ occur for low bias currents but this has the disadvantage that $g$ is a rapidly changing function of $\Phi_{x}$, making the adjustment of the working point critical. In contrast, for higher bias currents the functional form is smoother (see figure 7) and working point selection is easier and more stable.

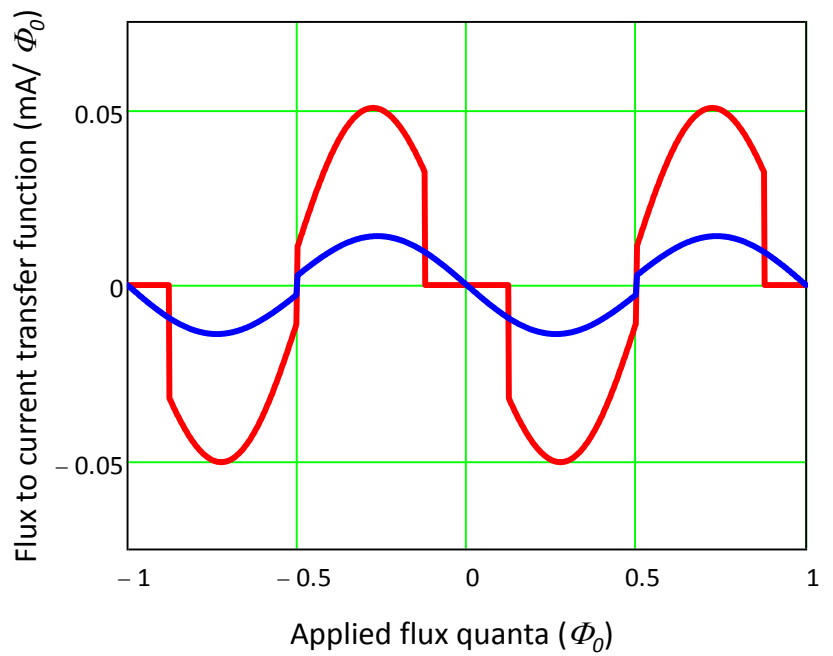

Fig. 7. Plot of transfer function $g$ for the same SQUID shown in fig. 6 for two different values of bias current a) $14 \mu \mathrm{A}$ (red curve) and b) $55 \mu \mathrm{A}$ (blue curve).

The results of this section demonstrate that, to overcome the input current noise of the SSA preamplifier, the flux to current transfer function $g=d I_{s} / d \Phi_{x}$ must be maximized, requiring a nanoSQUID with maximum critical current (without hysteresis) but also maximum $\Delta I$. For the SSA input current noise level quoted above, of $10 \mathrm{pA} / \mathrm{Hz}^{1 / 2}$ a minimum $I_{c 0}$ of $50 \mu \mathrm{A}$ and a value of $\Delta I>0.4$ would provide an SSA contribution to the input flux noise in the white noise region of the nanoSQUID of no more than $10^{-7} \Phi_{0} / \mathrm{Hz}^{1 / 2}$.

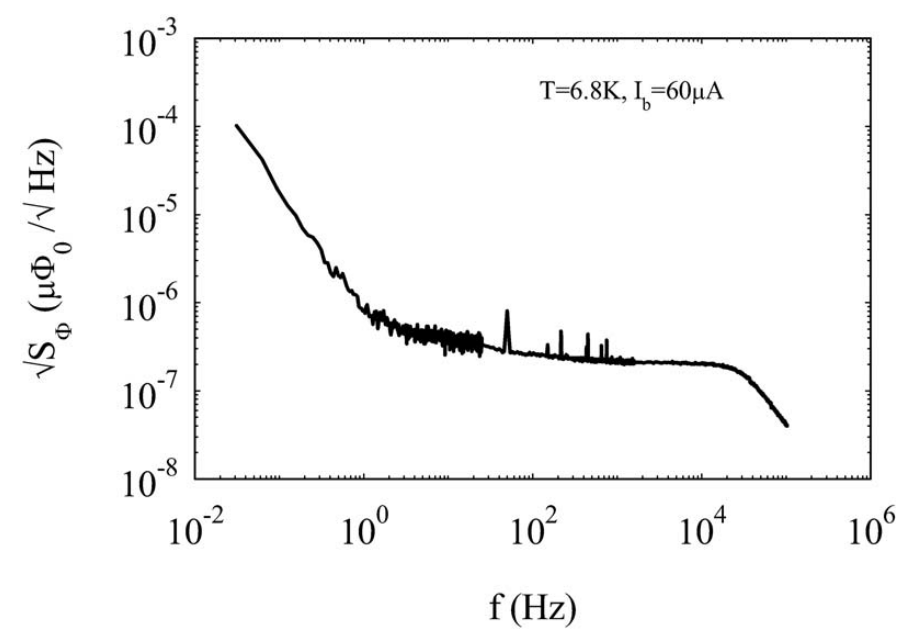

Fig. 8 Flux noise spectral density $\mathrm{S}$ vs frequency $\mathrm{f}$ at $\mathrm{T}=6.8 \mathrm{~K}$ with bias current of $60 \mu \mathrm{A}$ (see ref. [6]).

\section{MEASURED NOISE WITH SSA}

The noise output from a $\mathrm{Nb}$ nanoSQUID with loop diameter $350 \mathrm{~nm}$ and critical current of around $60 \mu \mathrm{A}$ has been measured using the SSA described above, at a temperature of 6.8K. Fig. 8 shows a plot of the observed flux noise power spectral density, referred to the nanoSQUID input, as a function of frequency. White noise dominates above around $1 \mathrm{kHz}$. Note also that the noise at lower frequencies does not follow a $1 / f$ dependence.

\section{SQUID LOOP INDUCTANCE AND ENERGY SENSITIVITY}

A key performance measure for nanoSQUIDs is the energy sensitivity $\varepsilon_{n}$. The relationship between the power spectral density of flux noise and the energy sensitivity is shown by the equation

$$
\varepsilon_{n}=\frac{\Phi_{n}^{2}}{2 L}
$$

where $L$ is the SQUID loop geometric inductance. This allows an individual SQUID's performance to be compared with that of any other similar device by referring the minimum detectable change measurable at the input terminal of the SQUID.

NanoSQUIDs are often used in a situation where there is no input flux transformer, instead the magnetic flux to be measured is applied directly to the SQUID loop, inductance $L$. The self-inductance of a nanoSQUID is clearly small, given their small size and we estimate for our devices as low as a few $\mathrm{pH}$. For small SQUIDs the kinetic inductance associated with the kinetic energy of the superconducting carriers cannot be ignored. This inductance adds to the geometric term to increase the $\beta_{L}$ parameter and therefore reduces the $\Delta I$ value. The kinetic inductance scales with the penetration depth of the superconducting film from which the SQUID loop is made. Fortunately our $\mathrm{Nb}$ thin films are relatively thick (100$200 \mathrm{~nm}$ ) and the penetration depth at the operating temperature is less than $\sim 50 \mathrm{~nm}$. This means that the total inductance should be no more than $20 \mathrm{pH}$. From the measured white noise value of $\Phi_{n} \sim 2.10^{-7} \Phi_{0} / H z^{1 / 2}$ the expected value for $\varepsilon_{n}$ is $\sim 4.10^{-33} \mathrm{~J} / \mathrm{Hz}$. Conventional quantum mechanical arguments suggest that the minimum value of $\varepsilon_{n}$ is $\hbar$ where $\hbar$ is Planck's constant divided by $2 \pi$. The measured value for our nanoSQUIDs currently correspond to $\varepsilon_{n} \sim 40 \hbar$

\section{MAGNETIC NANOPARTICLE DETECTION WITH NANOSQUIDS}

An important potential application of nanoSQUIDs is the detection and measurement of the properties of ever-smaller magnetic particles. This could be of significance not only for the IT and telecom communities (where complex spintronics properties may play an essential role in future developments) but also for future medical and biological applications. The burgeoning field of quantum technologies, including quantum information processing is increasingly relying on nanoSQUIDs for interrogation of single spin systems [15]. It was proposed some years ago that, if a small enough SQUID 
is used, its sensitivity should be sufficient to detect the reversal of a single Bohr magneton moment [16]. This can be seen as a grand challenge for nanoSQUIDs which has not been achieved in anything other than a prototype system at the time of writing [17].

\section{A. Coupling Between Nanoparticle and SQUID}

The significance of nanoscale SQUIDs for magnetic particle detection lies with the realisation that the sensitivity of a SQUID to the magnetisation of a particle improves as the SQUID loop size decreases towards the size of the particle. To estimate the flux coupled from a spherical magnetic particle with magnetisation $\boldsymbol{m}$ into a SQUID loop we make the assumption that the total magnetic moment is concentrated at the centre of the sphere. Then a simple dipolar magnetic field distribution may be numerically integrated across the area of the SQUID loop to find the magnetic flux coupled to the SQUID.

In agreement with the results of others [18,19] our modelling results show that the coupling between particle and SQUID is strongly dependent on the vertical distance above the SQUID loop, falling off rapidly as the dipole is moved outside the radius of the loop. Since SQUIDs are not absolute magnetometers it is only changes in the particle's magnetisation vector which will change the signal at the SQUID output. Such a change may be produced by changing the magnitude or direction of any applied polarising field. For a particle which possesses a net magnetic moment which is stable over a timescale greater than the inverse measurement bandwidth of the SQUID, any variation in the component of the resulting moment in the direction perpendicular to the SQUID loop will cause the SQUID readout voltage to change. Note, however, that the applied field will also contribute a component to the flux coupled to the SQUID. It is possible to calibrate out this effect as shown elsewhere [15], as the system is measured before and after the particle is attached.

In figure 9 we show calculations of the flux coupled into a nanoSQUID $200 \mathrm{~nm}$ loop size from a single Bohr magneton with moment oriented in the plane of the SQUID loop as it is translated across the centre of the loop.

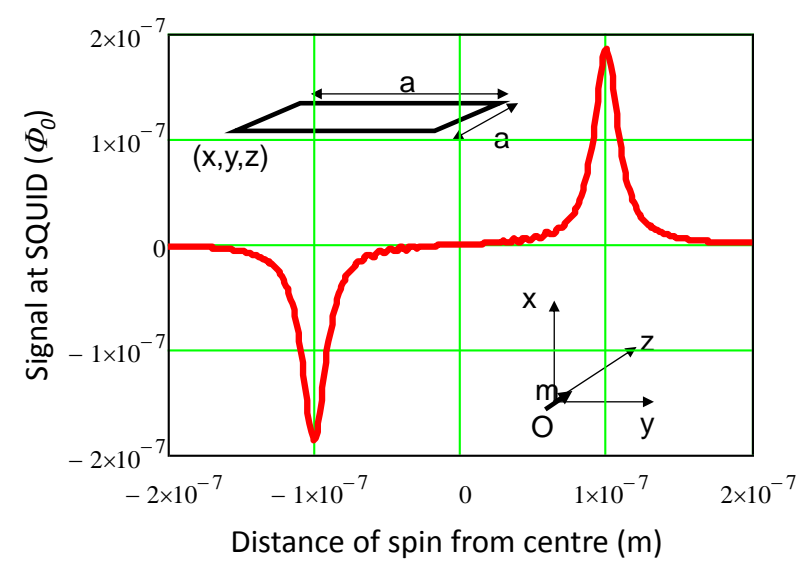

Fig. 9. Calculation of flux signal coupled into a square SQUID loop $200 \mathrm{~nm}$ on side by a single Bohr magneton moment aligned in SQUID plane as it is scanned across the loop at a height of $5 \mathrm{~nm}$. The inset shows the schematic of the axes and the moment.
The signal is a maximum as the moment coincides with the loop perimeter. The vertical distance for this calculation between moment and loop was set at $5 \mathrm{~nm}$, a realistic approach distance for a moment attached to an AFM cantilever, for example. The peak signal is around $10^{-7} \Phi_{0}$. Note that the SQUID noise shown in Fig. 8 suggests that the white noise figure achieved there is already within a factor of $2-3$ of detecting a single electronic spin within a $1 \mathrm{~Hz}$ bandwidth.

\section{OTHER NANOSQUID APPLICATIONS}

\section{A. Nanoelectromechanical system resonators and nanoSQUIDs}

As well as magnetic flux or field detection SQUIDs have already demonstrated a wide range of other extreme sensitivity sensing applications, for example physical displacement. The simplest approach is to couple the moving entity (which should be conducting or superconducting) directly to a SQUID loop, or indirectly through a flux transformer. The SQUID loop inductance will be affected by relative movement between it and the displaced item, thereby changing the output signal. Displacement sensing using macro SQUIDs has been demonstrated extensively [20,21]. It is timely to make use of nanoSQUIDs to read out the motion of another important new device the nanoelectromechanical system (NEMS). The combination of high sensitivity at the nanoscale with broad bandwidth makes a nanoSQUID an ideal sensor for high frequency NEMS resonators. A number of different arrangements have already proved capable of detecting the quantum ground state of a mechanical resonator, at frequencies ranging from $10 \mathrm{MHz}$ to $6 \mathrm{GHz}$ [22.23], all of which are potentially within the range of a nanoSQUID.

A crucial factor in the optimization of sensitivity of SQUID detection of the NEMS resonator movement hinges on the positioning of the two elements which should be as closely separated as possible [24]. The NPL fabrication process is as follows. A sharpened carbon fibre, fixed to a micromanipulator within a dual-beam FIB/SEM system, is attached to one corner of the frame supporting a mechanical resonator, using the e-beam to decompose organic source of carbon injected from a gas injection system (GIS). This produces a 'weld' of amorphous carbon.

The resonator itself (in the example here an $\mathrm{Al}$ coated $\mathrm{Si}$ beam, $1 \mu \mathrm{m}$ in length and $100 \mathrm{~nm}$ wide) is milled using a FIB and back-etched to a depth of $\sim 100 \mathrm{~nm}$ from the lower surface of its frame, allowing free movement above the SQUID loop when attached. The frame is aligned with the SQUID loop and secured on amorphous carbon pillars on either side. Lastly electrical connections to the resonator are made using $\mathrm{W}(\mathrm{CO})_{6}$ ), a conducting metallo-organic, introduced via the GIS. The slot SQUID, with rectangular loop designed to couple tightly to the beam resonator is shown in Fig 10a) and the resonator and SQUID during assembly in Fig. 10b). 
[Type text]

(a)

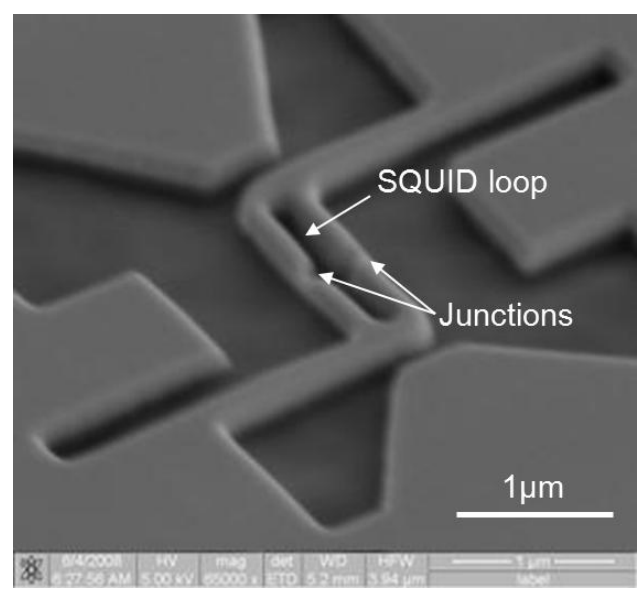

(b)

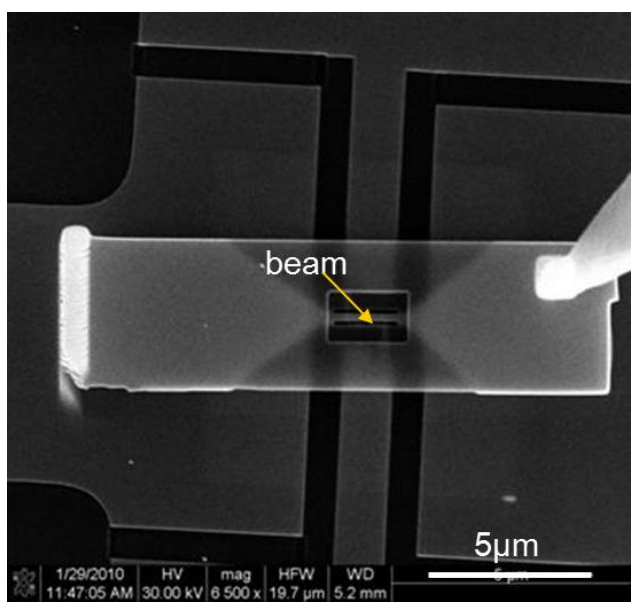

Fig.10. SEM images of (a) the slot-shaped nanoSQUID structure $(100 \mathrm{~nm} \times$ $900 \mathrm{~nm}$ ), and (b) the same SQUID coupled to a double-clamped beam.

Further ahead we plan to integrate the mechanical resonator directly within the nanoSQUID's loop, extending to the nanoscale some work described in ref. [25].

\section{B. Single Particle Detection using nanoSQUIDs}

Single photon detection using SQUIDs of conventional size has been demonstrated using a number of different arrangements, with a voltage-biased superconducting transition edge sensor being read out by a current sensing SQUID. The impedance matching is particularly favourable for this arrangement and the technology has developed rapidly over recent years, culminating in the production and use of large arrays of multiplexed transition edge sensors (TES) sensors which have proved invaluable in observational astronomy. (For a recent review see Irwin and Hilton [26]).

Following the proposal and detailed development work on a variant of this idea by McDonald [27] NPL has developed a SQUID-based micro-calorimeter which detects the increase in temperature of a thin film superconducting absorber contained within the SQUID loop [28-30]. The absorber is biased below its transition temperature such that on absorbing energy from a photon the rapid change in superconducting penetration depth of the absorber at a temperature slightly below $T_{c}$ gives rise to a change in the effective loop area and therefore a change in the SQUID output signal. Because the absorber is held just below $T_{c}$ it is not subject to Johnson noise which sets a limit on the sensitivity of conventional TES detectors. We have shown that this is able to measure the deposited energy from single optical photons with a resolution of at least $0.1 \mathrm{eV}$ [5].

To achieve adequate quantum efficiency for capture of photons the capture device should have a linear dimension at least comparable to the wavelength of the radiation, implying that for visible or lower energy photons the absorber should be around $1 \mu \mathrm{m}$ or more. However for higher energy photons (e.g. X-rays) or massive particles absorbers coupled to nanoSQUIDs provide a way of potentially improving the spatial resolution of TES imaging systems. Following initial work on a SQUID based ion beam dosimetry detector [31] aimed at providing a traceable dosimeter for proton irradiation we are also working on similar detection methods for future high resolution 'nanobeam' measurements.

\section{CONCLUSIONS AND Future WORK}

NanoSQUIDs are a recent development of a superconducting technology which is already 50 years old. We have attempted to demonstrate in this paper that development of Dayem bridge junctions for thin film SQUIDs has opened up a new regime of low noise operation at relatively elevated temperatures. Simple deposition, fabrication and assembly techniques can provide a very wide range of different geometries and transduction methods for ultra-sensitive measurement. Furthermore, controlled exposure of the SQUID junctions using the FIB can lead to suppression of the Tc and therefore extended operation over a wider temperature range. Use of different materials, combined with electron beam lithography has also led to the demonstration of Dayem bridge nanoSQUIDs which operate at sub-1 $\mathrm{K}$ temperatures [32]. As a result of these developments and those made at other laboratories a wealth of innovative applications of nanoSQUIDs is on the horizon, with the promise of other innovations still to come. The impact of analogue detectors on quantised systems promises a sensor revolution in many ways. Higher resolution spectroscopy of photons over a wider frequency range, detection of single electronic spin flips and the observation of the quantum mechanical ground state of a mechanical resonator are all measurement challenges which seem to lie within the future capablilities of nanoSQUIDs.

\section{ACKNOWLEDGMENT}

We thank Dr. T. Schurig and colleagues at the PTB (Berlin) for helpful technical collaborations and discussions, and for providing the SQUID series array amplifier used in our nanoSQUID noise measurements. The work reported here was supported in part by the EMRP projects 'MetNEMS' NEW-08 and 'BioQUART'SIB-06. The EMRP is jointly funded by the EMRP participating countries within EURAMET and the European Union. 


\section{REFERENCES}

[1] J. Clarke and A. Braginski, 'The SQUID Handbook', Wiley-VCH Verlag (2005)

[2] C P Foley and H Hilgenkamp, 'Why nanoSQUIDs are important:', Supercond. Sci. Technol. Vol. 22 064001(2009)

[3] M. Jamet, W. Wernsdorfer, C. Thirion, D. Mailly, 'Magnetic Anisotropy of a Single Cobalt Nanocluster', Phys. Rev. Lett. Vol. 86 pp. 4676-9 (2001)

[4] S K H Lam and D L Tilbrook, 'Development of a niobium nanosuperconducting quantum interference device for the detection of small spin populations', Appl. Phys. Lett. Vol. 82 pp. 1078-80 (2003)

[5] L Hao, "Quantum Detection Applications of NanoSQUIDs fabricated by Focused Ion Beam", J. Phys.: Conf. Ser. 286, 012013, 2011.

[6] L. Hao, J. C. Macfarlane, J. C. Gallop, D. Cox, J. Beyer, D. Drung, and T. Schurig, "Measurement and noise performance of nanosuperconducting-quantum interference devices fabricated by focused ion beam", Appl. Phys. Lett., vol. 92, p. 192507, 2008.

[7] R. Wölbing, J. Nagel, T. Schwarz, O. Kieler, T. Weimann, J. Kohlmann, A. B. Zorin, M. Kemmler, R. Kleiner and D. Koelle, 'Nb nano superconducting quantum interference devices with high spin sensitivity for operation in magnetic fields up to 0.5 T', Appl. Phys. Lett. vol. 102 (2013) 192601

[8] Carmine Granata, Antonio Vettoliere, Roberto Russo, Matteo Fretto, Natascia De Leo, and Vincenzo Lacquaniti, 'Three-dimensional spin nanosensor based on reliable tunnel Josephson nanojunctions for nanomagnetism investigations', Appl. Phys. Lett. vol.103 (2013) 102602

[9] See http://www.srim.org/

[10] W. J. Skocpol, M. R. Beasley, and M. Tinkham, 'Self-heating hotspots in superconducting thin-film microbridges', J. Appl. Phys. 45, 4054 (1974).

[11] D. Hazra, L. M. A. Pascal, H. Courtois, and A. K. Gupta, 'Hysteresis in superconducting short weak links and $\mu$-SQUIDs', Phys. Rev. B 82, 184530 (2010).

[12] S. Rozhko, T. Hino, A. Blois, L. Hao, J. C. Gallop, D. C. Cox, and E. J. Romans, 'Study of Low-Frequency Noise Performance of NanobridgeBased SQUIDs in External Magnetic Fields', IEEE Trans. Appl. Supercon., 23, 1601004 (2013)

[13] D. Drung, C. Aßmann, J. Beyer, A. Kirste, M. Peters, F. Ruede, and T. Schurig, IEEE Trans. Appl. Supercond. Vol. 17, 699 (2007).

[14] E. J. Romans, S. Rozhko, L. Young, A. Blois, L. Hao, D. Cox, J. C. Gallop, "Noise Performance of Niobium Nano-SQUIDs in Applied Magnetic Fields", IEEE Trans. Appl. Supercond., vol.21, pp. 404-7, 2011.

[15] L. Hao, C. Aßmann, J. C. Gallop, D. Cox, F. Ruede, O. Kazakova, P. Josephs-Franks, D. Drung, and Th. Schurig, 'Detection of single magnetic nanobead with a nano-superconducting quantum interference device', Appl. Phys. Lett. Vol. 98, 092504 (2011).

[16] J. Gallop, Supercond. Sci. Technol. 16, 1575 (2003)

[17] J. A. Sidles, J. L. Garbini, K. J. Bruland, D. Rugar, O. Züger, S. Hoen, and C. S. Yannoni, 'Magnetic resonance force microscopy', Rev. Mod. Phys. Vol. 67 p249 (1995)

[18] S K H Lam, Wenrong Yang, H T R Wiogo and C P Foley, 'Attachment of magnetic molecules on a nanoSQUID', Nanotechnology vol. 19 285303 (2008).

[19] S K H Lam and D L Tilbrook, 'Development of a niobium nanosuperconducting quantum interference device for the detection of small spin populations', Appl. Phys. Lett. Vol. 82 pp. 1078-80 (2003)

[20] M. Moody, Ho Jung Paik and Edgar R. Canavan, 'Three-axis superconducting gravity gradiometer for sensitive gravity experiments', Rev. Sci. Instrum. Vol. 73, 3957 (2002)

[21] H J Paik, 'Superconducting tunable-diaphragm transducer for sensitive acceleration measurements', J. Appl. Phys. Vol. 47, 1168 (1976)

[22] A. D. O'Connell, M. Hofheinz, M. Ansmann, Radoslaw C. Bialczak, M. Lenander, Erik Lucero, M. Neeley, D. Sank, H. Wang, M. Weides, J. Wenner, John M. Martinis \& A. N. Cleland, "Quantum ground state and single-phonon control of a mechanical resonator", Nature vol. 464, pp. 697-703, (2010).

[23] J. D. Teufel, T. Donner, Dale Li, J. W. Harlow, M. S. Allman, K. Cicak, A. J. Sirois, J. D. Whittaker, K. W. Lehnert \& R. W. Simmonds, "Sideband cooling of micromechanical motion to the quantum ground state", Nature vol. 475, pp. 359-63 ( 2011).

[24] L. Hao, D.C Cox, J.C. Gallop, J. Chen, S. Rozhko, A. Blois and Ed. Romans, 'Coupled NanoSQUIDs and Nano-Electromechanical Systems
(NEMS) Resonators', IEEE Trans. Appl. Supercond., 23(3), 1800304 (2013).

[25] S. Etaki, M. Poot, I. Mahboob, K. Onomitsu, H. Yamaguchi, and H. S. J. van der Zant, "Motion detection of a micromechanical resonator embedded in a d.c. SQUID," Nat. Phys. vol. 4, pp. 785-788, 2008.

[26] K D Irwin, G C Hilton in' Transition-Edge Sensors' in Cryogenic Particle Detection' Ed: C. Enss, Vol. 99, pp 63-149 (2005).

[27] D G McDonald, 'Novel superconducting thermometer for bolometric applications', Appl. Phys. Lett. Vol.50 pp.775-7 (1987)

[28] L. Hao, et al., 'Inductive superconducting transition-edge photon and particle detector', IEEE Trans. Appl. Supercond., vol. 13, pp.622 -625 (2003)

[29] L. Hao, et al., 'Inductive superconducting transition-edge detector for single-photon and macro-molecule detection', Supercond. Sci and Technol., vol. 16, pp.1479 -1482 2003

[30] L. Hao, J. C. Macfarlane, S. K. H. Lam, C. P Foley, P. Josephs-Franks, and J. C. Gallop, 'Inductive Sensor Based on Nano-Scale SQUIDs', IEEE Trans. Appl. Supercond., vol. 15, 514-6 (2005)

[31] S. Galer, L. Hao, J. Gallop, H. Palmans, K. Kirkby and A. Nisbet, 'Design concept for a novel SQUID based microdosimeter', MICROS 2009 Symposium Proceedings, Special issue of Radiation Protection Dosimetry, Oxford Journals , Oxford University Press (2010).

[32] A. Blois, S. Rozhko, L. Hao, J. C. Gallop and E. J. Romans, 'Proximity effect bilayer nano superconducting quantum interference devices for millikelvin magnetometry.' J. Appl. Phys. Vol. 114, 233907 (2013). 


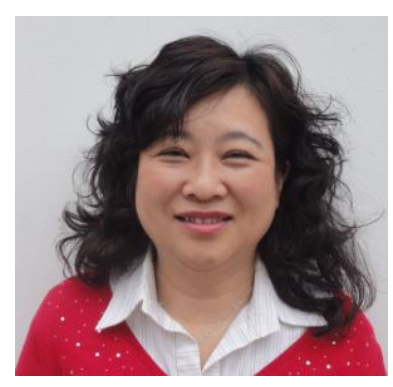

Ling Hao received the BSc degree in general physics and the MSc degree in solid state physics from Beijing Normal University, China. She moved to the UK in 1992 and received the $\mathrm{PhD}$ degree at the Department of Physics and Applied Physics, University of Strathclyde, UK, for research on electronic noise in superconducting devices in 1995. Since then she has worked at NPL. She has published more than 100 papers in refereed journals and four book chapters. Ling is also a visiting professor at Imperial College and a member of the Superconductivity Committee of the Institute of Physics. She is leading work on applications of nanoscience, superconducting electronics and microwave technology for precision measurements, aimed at single particle measurements and metrology with nanoSQUIDs and nanoelectromechanical system (NEMS) resonators. She is also working on low dimensional carbon, including carbon nanotubes and graphene transport measurements. Ling is a Fellow of the Institute of Physics (UK) and a Chartered Physicist.

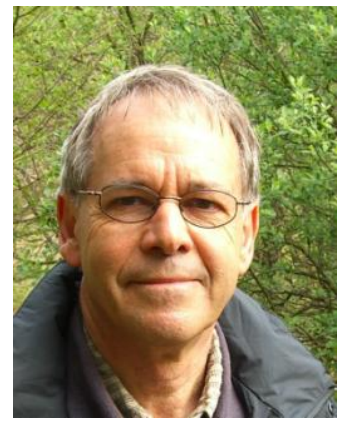

John Gallop received his B.A (1965) and D.Phil. (1970, in ultra low temperature physics) from Oxford University. He joined NPL in 1969 and worked on metrology applications of macroscopic quantum aspects of superconductivity (involving fundamental constant determinations, SQUIDs and high frequency applications) for a number of years. Later he spent more than a decade on research into the applications of high temperature superconductivity. He is an emeritus senior NPL fellow and was head of the Quantum Detection Group until retirement in 2003. He is currently researching developments in nanoscience, including transport properties of graphene and carbon nanotubes, single particle measurements with nanoSQUIDs, sub-micron Hall sensors and the metrology of nanoelectromechanical (NEMS) resonators. John is a Fellow of the UK Institute of Physics.

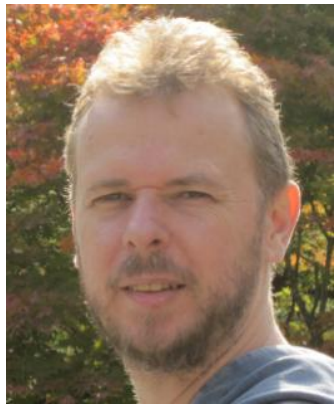

David Cox received his M.Sc from Birmingham University and a Ph.D from Cambridge University (2001) and is now a senior research fellow at the University of Surrey. He has been seconded full-time to the National Physical Laboratory as a senior research scientist since 2005, primarily for his expertise in the area of Focused Ion Beam technology. He has worked with most divisions across NPL in this time, but is largely associated with the Quantum Detection and Materials groups. He has published over 80 papers in several research fields ranging from single crystal superalloys to thermal transport in carbon nanotubes. He is currently working mainly on applications of focused ion beam to superconducting devices.

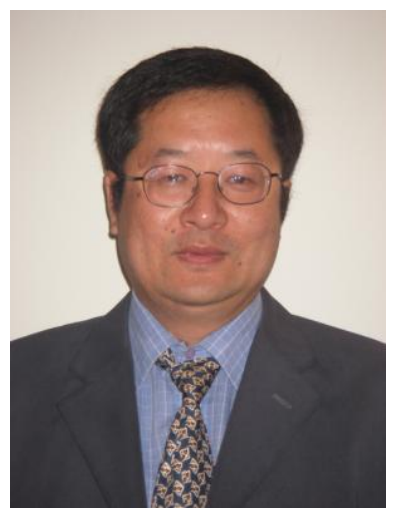

Dr Jie Chen received his BEng (1984) and MSc (1987) from Beihang Univ., and a DPhil (1995) from the University of York. He has been a Lecturer in Brunel University since February 1998. Before that, he worked in The University of Hull, University of Strathclyde and University of York as a lecturer/research associate. His main field of expertise is on robust control systems, fault diagnosis and faulttolerant control, with emphasis on industrial applications such as aircraft control system and automotive control systems. Dr Chen also has research experience on multiphysics modelling of nano-scale sensing devices and he is very experienced in applying nonlinear system modelling techniques to thermal and environmental systems. He has published over 60 papers and was awarded a best journal paper prize in 1997 by the IET/IEE. His research book on fault diagnosis for dynamic systems has attracted more than 2,700 citations. He is a member of the IFAC Technical Committee SAFEPROCESS and an associate editor of Int. J. of Sys. Sci. 